\section{O Instituto Aggeu Magalhães e sua figura símbolo: Frederico Simões Barbosa}

\author{
The Aggeu Magalhães Institute and its symbolic \\ figure: Frederico Simões Barbosa
}

\author{
El Instituto Aggeu Magalhães y su figura símbolo: \\ Frederico Simões Barbosa
}

1 Centro de Pesquisas Aggeu Magalhães, Fundação Oswaldo Cruz, Recife, Brasil.

Correspondência S. P. Brandão Filho Centro de Pesquisas Aggeu Magalhães, Fundação Oswaldo Cruz.

Av. Prof. Moraes Rego $s / n$, Recife, PE 50670-420, Brasil. sinval@cpqam.fiocruz.br
Na década de 1940, a maioria dos brasileiros, sobretudo no Nordeste, vivia em regiões rurais e as doenças mais preocupantes eram a esquistossomose, as leishmanioses, a tripanossomose americana (doença de Chagas) e outras doenças infecciosas de grande prevalência, as Grandes Endemias. Relatórios de viagens de cientistas do Instituto Oswaldo Cruz (IOC), por meio de textos e fotos, revelaram "uma população doente e abandonada pelo governo” 1 (p. 13-4), justificando a expressão usada por Miguel Pereira, segundo a qual o Brasil de então era "um imenso hospital".

Esses relatórios evidenciaram a necessidade de realização de estudos que aprofundassem os conhecimentos sobre a realidade sanitária do país. Em 1938, após a constatação da elevada prevalência e graves consequências da esquistossomose e outras endemias parasitárias na população nordestina, foi estabelecida uma parceria entre o IOC, representado pelo Superintendente do Serviço de Estudos das Grandes Endemias, Dr. Evandro Chagas, e o Estado de Pernambuco, representado por um grupo de professores da Faculdade de Medicina do Recife, liderado pelo professor Aggeu de Godoy Magalhães. Nessa ocasião, foi proposta a elaboração de um plano de trabalho para estudos conjuntos de medicina social, criando-se a Comissão de Estudos de Patologia Experimental do Nordeste.

Essa Comissão foi pioneira nos estudos sobre a esquistossomose, chegando a publicar, em
Eridan M. Coutinho 1

Sinval P. Brandão Filho ${ }^{1}$

1940, nas Memórias do Instituto Oswaldo Cruz, substancioso trabalho de 78 páginas, com várias ilustrações, versando sobre aspectos da epidemiologia, clínica e anatomia patológica da endemia 2 . Pode-se considerar, então, que essa iniciativa inspirou posteriormente o então Ministério da Educação e Saúde a criar em Pernambuco um Instituto de Pesquisas dedicado ao estudo da esquistossomose e outras helmintoses. O trabalho realizado por Pellon\&Teixeira na década de 19403, determinando a distribuição geográfica e a prevalência da esquistossomose no Brasil, quando elevadas taxas de infecção foram detectadas em Pernambuco e em outras áreas do país alarmaram a comunidade científica e as autoridades de saúde. Ao mesmo tempo, a falta de conhecimentos básicos sobre a taxonomia, a biologia e a ecologia do Schistosoma mansoni e seus transmissores no Brasil dificultava a elaboração de programas efetivos de controle da endemia.

Em 1948, veio a Pernambuco o sanitarista Amilcar Barca Pellon, diretor da Divisão de Organização Sanitária do Departamento Nacional de Saúde (Ministério da Educação e Saúde), para tratar da instalação de um futuro Centro de Estudos em Endemias Rurais e Doenças Parasitárias no Recife, a ser construído em terreno cedido pelo então Governador de Pernambuco Dr. Alexandre Barbosa Lima Sobrinho, situado na Rua do Espinheiro 106, no bairro de igual nome, sendo, então, iniciada a construção pelo Governo Federal. Todavia, a conclusão dessa obra não 
Reunião realizada nas dependências do Instituto de Pesquisas Aggeu Magalhães (década de 1960) (Acervo particular de Eridan Coutinho). Da esquerda para direita, os pesquisadores: Aggeu Magalhães Filho, Jandira de Moraes Barbosa, Ivete de Melo Barbosa, Eridan Coutinho, Frederico Simões Barbosa, Guilherme Montenegro Abath, Bento Magalhães Neto. Na parede, o quadro de Amílcar Barca Pellon, chefe da Divisão de Organização Sanitária (DOS) do Ministério da Saúde.

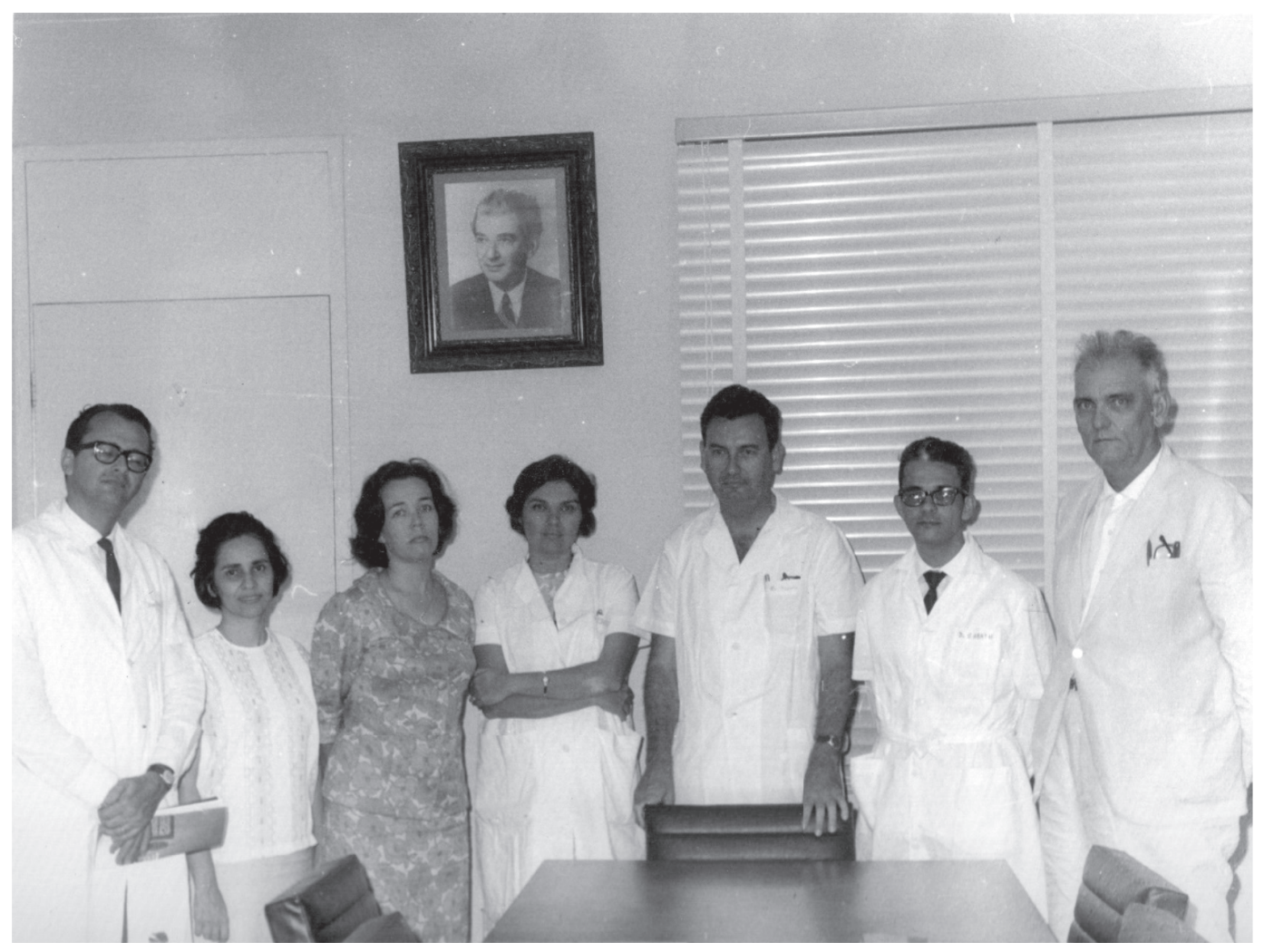

foi presenciada pelo Prof. Aggeu Magalhães, que provavelmente seria indicado para dirigi-la, devido ao seu falecimento em 31 de julho de 1949, um ano antes da inauguração do Instituto que hoje leva seu nome.

Concebido, inicialmente, como o "primeiro centro de pesquisas organizado no país para estudo exclusivo das questões ligadas aos nossos problemas de helmintoses" (Barca Pellon - discurso de inauguração proferido em 2 de setembro de 1950), coube ao jovem médico sanitarista Frederico Adolfo Simões Barbosa, então com 34 anos de idade, a tarefa de organizar o recém-inaugurado Instituto Aggeu Magalhães, não somente do ponto de vista administrativo como também de suas atividades científicas iniciais.

Nascido em Recife em 27 de julho de 1916, filho e neto de conceituados médicos clínicos pertencentes à tradicional família de Pernambuco, graduou-se em Medicina pela Faculdade de Medicina do Recife (atual Universidade Federal de Pernambuco) em dezembro de 1938, tornando-se doutor em medicina por esta Escola em 1943. Vocacionado para a pesquisa, fez estudos de pós-graduação na Universidade de São Paulo em 1939, sob a supervisão dos professores Samuel B. Pessoa (Parasitologia) e Floriano de Almeida (Micologia). Em 1945, viajou para os Estados Unidos, onde obteve o diploma de "Master in Public Health" pela Faculdade de Higiene e Saúde Pública da Universidade Johns Hopkins, estagiando, ainda, no Museu Nacional de Washington e na Universidade de Michigan, nas áreas de Entomologia e Malacologia. Nesse período no exterior ampliou ainda mais o seu interesse científico para o campo das ciências 
biológicas e, após voltar ao Brasil, graduou-se também em História Natural e obteve os títulos de docência livre em Parasitologia (1942), Microbiologia (1950), Zoologia (1953) e Medicina Preventiva (1960), todos pela Universidade Federal de Pernambuco, onde tornou-se também Professor Titular concursado de Higiene e Medicina Preventiva (1968). Em sua extensa e brilhante carreira no país, foi também Professor Titular de Medicina Comunitária da Universidade de Brasília, 1972-1981, Professor de Medicina Preventiva da Universidade Federal de São Carlos, 1982, e Professor Titular concursado de Epidemiologia da Escola Nacional de Saúde Pública, Fundação Oswaldo Cruz, Rio de Janeiro, 1983, onde lecionou e liderou diversos programas e iniciativas inéditas, entre elas a criação dos Cadernos de Saúde Pública. Com sua liderança de Scholar, formou diversas gerações de pesquisadores, orientando inúmeras teses e atraindo com sua liderança pesquisadores de diversas origens e formações, estimulando ao mesmo tempo em sua longa trajetória o intercâmbio com outras instituições científicas do Brasil e do exterior.

Fiel ao objetivo central de sua criação, Frederico fez da esquistossomose o alvo principal de suas investigações no Instituto Aggeu Magalhães, considerando a importância da doença para todo o Nordeste, por sua elevada prevalência, expressivas taxas de morbidade e mortalidade e repercussões socioeconômicas para a região; uma época áurea para a instituição, para a qual contribuiu o seu primeiro núcleo de pesquisadores. Reconhecido como exímio rastreador de vocações e caçador de talentos, não foi difícil ao Professor Frederico adicionar jovens estudantes ao núcleo inicial de pesquisadores do Instituto Aggeu Magalhães, que aos poucos se incorporaram às diversas equipes de trabalho, como foi o caso dos acadêmicos de medicina Marcello de Vasconcelos Coelho e Eridan de Medeiros Coutinho, dos estudantes de biologia Célio Almeida e Alzira Almeida, dentre outros, que futuramente foram integrados à instituição.

O impacto produzido por pesquisas pioneiras, tais como ensaios com diferentes moluscicidas para o controle do caramujo transmissor da esquistossomose, estudos acerca da taxonomia, biologia, bioquímica e ecologia de moluscos, pesquisas sobre hospedeiros naturais do $S$. mansoni e seu papel na transmissão do parasito, diferentes aspectos da patologia experimental e humana e suas relações com a desnutrição na esquistossomose mansônica, além de alguns trabalhos iniciais sobre filariose linfática, projetaram o Instituto Aggeu Magalhaes no cenário científico do Brasil e do exterior. Sob a esclarecida direção do Professor Frederico Simões Barbo- sa, a comunidade científica passou a reconhecer a importância do Instituto Aggeu Magalhães, situado no esquecido nordeste brasileiro, intensificando-se, ano após ano, as visitas e os estágios de outros pesquisadores, incluindo figuras de destaque no cenário científico internacional, tais como Frank Hawking (National Institute for Medical Research - Londres, Inglaterra), Tulio Pizzi (Universidade do Chile, Chile), Emile Malek (Estados Unidos), Irving Fox, entre outros.

Desde os primórdios de sua atuação, tendo como diretor o Professor Frederico, o Instituto Aggeu Magalhães - mais tarde, Centro de Pesquisas Aggeu Magalhães (CPqAM) - já contribuía para a formação de recursos humanos para os quadros do Ministério da Saúde, das Secretarias Estaduais e Municipais de Saúde e das universidades do Nordeste, por meio da formação de pesquisadores, professores de nível superior e técnicos de laboratório, mediante estágios e cursos de especialização ministrados por seu corpo técnico. A partir de 1962, as ações de pesquisa no CPqAM começaram a sofrer diversificação, datando desta época os primeiros estudos realizados sobre peste, leishmaniose e filariose, além da esquistossomose, tendo sido inaugurada em 1966, no Município de Exu (Pernambuco), uma Estação de Campo destinada a pesquisas sobre focalização e epizootização da peste e fatores responsáveis pela peste humana e de roedores, um amplo projeto elaborado por pesquisadores visitantes do Instituto Pasteur de Paris (França) e por consultores da Organização Pan-Americana da Saúde/Organização Mundial da Saúde (OPAS/OMS), com resultados que permitiram a introdução de importantes modificações nas atividades de vigilância, controle e diagnostico da peste no Ministério da Saúde. Datam ainda da gestão do Professor Frederico, importantes publicações sobre morbidade e evolução clínica da esquistossomose, problemas relacionados com o desenvolvimento da esquistossomose em áreas de irrigação da região semiárida do nordeste brasileiro, aspectos da epidemiologia e estratégias de intervenção em áreas endêmicas e repercussões sociais e econômicas da esquistossomose em Pernambuco. Após deixar a direção do CPqAM, o Professor Frederico exerceu atividades no exterior (1969-1971), como perito em doenças parasitárias da OMS em Genebra (Suíça), coordenando o programa de esquistossomose da OMS, assessorando e avaliando programas de pesquisa em vários países, sobretudo da África.

Durante a fecunda existência do Professor Frederico Simões Barbosa, sua abrangente formação profissional e humanística permitiu-lhe incursionar, como pesquisador e professor, em 


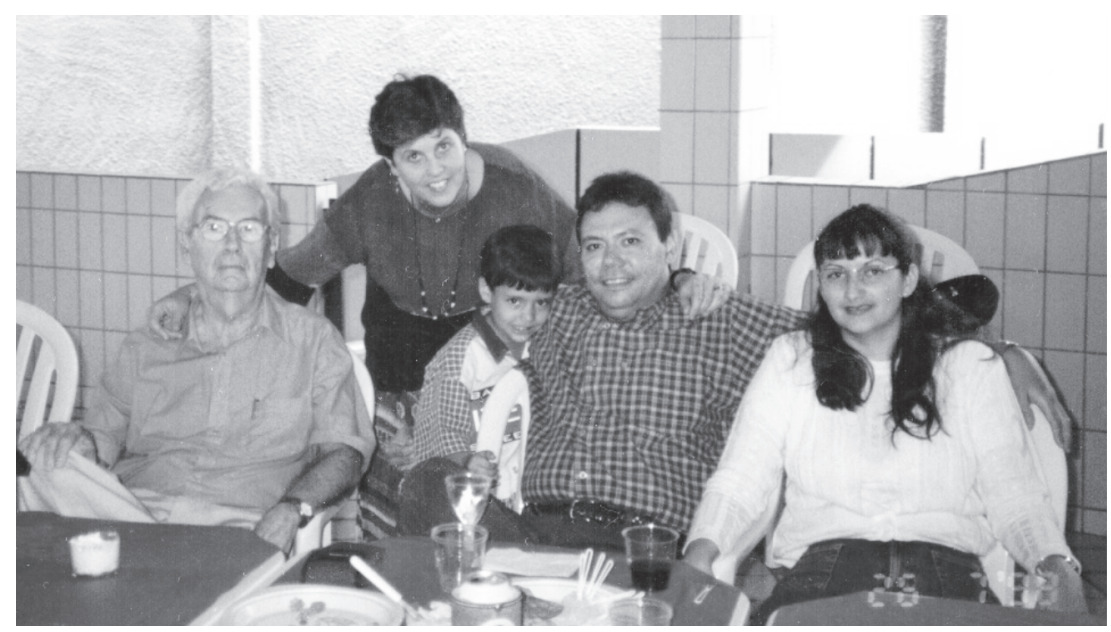

diferentes áreas da pesquisa biomédica, permitindo-lhe, posteriormente, migrar da área básica para a da saúde comunitária e medicina preventiva. Publicou centenas de artigos científicos, além de livros e capítulos de livros. Com sua liderança, foi um dos principais artífices e sócio-fundador da Sociedade Brasileira de Medicina Tropical em 1962, e da Associação Brasileira em Saúde Coletiva, em 1979. Como sanitarista, deixou importante contribuição, coordenando variados projetos de integração docente-assistencial realizados em Pernambuco, Brasília e Espírito Santo. Sua objetividade no trato das questões científicas está bem sintetizada quando, a propósito de estudos epidemiológicos a serem realizados em situações de limitações de vários tipos, dizia ser necessário, muitas vezes, "trocar a complexidade pela simplicidade, a extensão pela profundidade, a rígida e cega padronização técnica pela criatividade, a alienação por uma visão comprometida com a comunidade", combatendo a excessiva admiração pelas conquistas científicas produzidas no mundo desenvolvido e condenando os estudos repetitivos ou imitativos, por vezes tão frequentes.
Além de seus méritos intelectuais e profissionais, o Professor Frederico encantava pelo cavalheirismo, humanismo, generosidade, pelo estilo de vida simples e sensibilidade para com os problemas dos mais humildes. Também se destacava pela dedicação às instituições públicas às quais serviu, e pela atenção dispensada aos jovens estudantes que o procuravam para ouvir sua opinião sensata e experiente, revelando sempre interesse pelo seu crescimento científico. Quando diretor, costumava realizar visitas frequentes e informais aos diversos laboratórios do CPqAM, indagando sobre resultados e dificuldades encontradas na execução dos projetos e outras tarefas cotidianas de seus estudantes e pesquisadores, para os quais tinha sempre uma palavra de estímulo ou uma sugestão para solucionar os problemas.

Por todas essas características pessoais e pela sua valiosa contribuição científica à instituição que dirigiu com dedicação e eficiência durante 16 anos, gestão mais longa da história do CPqAM, pode ser considerado figura símbolo do Centro de Pesquisas Aggeu Magalhães, a Fiocruz Pernambuco. 


\section{Colaboradores}

Ambos os autores contribuíram igualmente na produção do artigo.
1. Magalhães Filho A, Klein L. Aggeu Magalhães: um pioneiro. Rio de Janeiro: Fundação Oswaldo Cruz; 2000.

2. Magalhães A, Coutinho B, Gouvêa L, Lucena D, Ignacio L. Estudos sobre a esquistossomose em Pernambuco, Brasil. Mem Inst Oswaldo Cruz 1940; 35:205-83.
3. Pellon AB, Teixeira I. Distribuição da esquistossomose mansônica no Brasil. Trabalho apresentado no 8o Congresso Brasileiro de Higiene. Rio de Janeiro: Divisão da Organização Sanitária; 1950. 